\title{
An in silico pipeline to filter the Toxoplasma gondii proteome for proteins that could traffic to the host cell nucleus and influence host cell epigenetic regulation
}

\author{
Genevieve Syn, Jenefer M Blackwell, Sarra E Jamieson, Richard W Francis/+ \\ University of Western Australia, Telethon Kids Institute, Subiaco, Western Australia, Australia
}

Toxoplasma gondii uses epigenetic mechanisms to regulate both endogenous and host cell gene expression. To identify genes with putative epigenetic functions, we developed an in silico pipeline to interrogate the T. gondii proteome of 8313 proteins. Step 1 employs PredictNLS and NucPred to identify genes predicted to target eukaryotic nuclei. Step 2 uses GOLink to identify proteins of epigenetic function based on Gene Ontology terms. This resulted in 611 putative nuclear localised proteins with predicted epigenetic functions. Step 3 filtered for secretory proteins using SignalP, SecretomeP, and experimental data. This identified 57 of the 611 putative epigenetic proteins as likely to be secreted. The pipeline is freely available online, uses open access tools and software with user-friendly Perl scripts to automate and manage the results, and is readily adaptable to undertake any such in silico search for genes contributing to particular functions.

Key words: Toxoplasma gondii - nuclear localisation - epigenetics

Toxoplasma gondii is a ubiquitous eukaryotic parasite associated with congenital or acquired ocular and/ or brain lesions. It can influence the host's transcriptome, including through modulation of the host epigenome (Leng and Denkers 2009, Leng et al. 2009, Lang et al. 2012). The ability of infectious disease organisms to modulate the host epigenome is an area of increasing interest, which is likely to underpin development of novel therapeutics (Vanagas et al. 2012, Syn et al. 2016). Experimental studies of $T$. gondii have demonstrated the presence of epigenetic machinery, including histone (Hettmann and Soldati 1999) and lysine (Wang et al. 2014) acetyltransferases, histone deacetylases (Vanagas et al. 2012), SET domain-containing histone lysine methyltransferases (Sautel et al. 2007), SWI/SNF2 proteins capable of influencing gene expression through ATP-dependent nucleosome remodelling (Sullivan Jr et al. 2003), and two predicted cytosine-5 DNA methyltransferase 2 (DNMT2) homologues (Wei et al. 2017).

Availability of pathogen genome sequences provides the opportunity to undertake a more global approach to identify proteins mediating epigenetic effects, particularly in the context of increasing public domain data and tools that facilitate filtering approaches to identify all genes involved in specific molecular pathways. Here we

doi: 10.1590/0074-02760170471

Financial support: RWF was supported by the Australian Medical

Bioinformatics Resource (AMBeR) Project under an NHMRC-funded Multiinstitutional Agreement (Project ID 404025), the WA State Government

Centres of Excellence Program, and the McCusker Charitable Foundation.

GS was supported by an Australian Postgraduate Award and the Stan and Jean Perron Top-Up Award.

+ Corresponding author: richard.francis@telethonkids.org.au

SEJ and RWF contributed equally to this work.

Received 31 October 2017

Accepted 17 April 2018 develop an in silico pipeline to first identify all proteins in the T. gondii genome that can both target to eukaryotic cell nuclei and have putative epigenetic functions, and secondly to filter for those which also have secretory signals that could allow them to exit the parasite and affect the host epigenome directly. The pipeline is freely available (http://bioinformatics.childhealthresearch.org.au/ software/nuc loc/), uses open access tools and software with user-friendly Perl scripts to automate and manage the results, and is readily adaptable to undertake any in silico search for genes contributing to specific functions.

The source data used here was ToxoDB (http://toxodb. org) (Gajria et al. 2007), which incorporates sequence and annotation from GenBank for the three clonal lineages of T. gondii: GT1 (Type I), ME49 (Type II) and VEG (Type III). We exported the T. gondii (ME49 strain) proteome of 8318 proteins. The filtering rationale (Figure) aimed to find all proteins predicted to: (i) go to a eukaryotic nucleus; (ii) have an epigenetic function; and (iii) be secreted from the parasite into the host cell. The pipeline and filtering parameters summarised below are described in more detail in the Supplementary data IV. Table I summarises the protein numbers from each step of the in silico pipeline. Supplementary data I contains both the raw and summary results arising from running all $8318 \mathrm{~T}$. gondii encoded proteins through all in silico tools.

To predict proteins with the potential to localise to eukaryotic cell nuclei, we first used PredictNLS (version 1.3) (Cokol et al. 2000) combined with AccPro (Magnan and Baldi 2014) to identify proteins containing an exposed putative nuclear localisation signal (NLS), and calculated the overall percentage of exposed residues for each NLS motif. Some proteins, whilst not themselves containing an NLS, gain access to the nucleus when bound to an NLS-containing protein. Proteins may also contain novel NLSs. To capture proteins such as these we used NucPred (version 1.1), a tool that predicts whether 
or not proteins spend time in the nucleus (Brameier et al. 2007). Of the $8318 T$. gondii encoded proteins extracted from ToxoDB, 3408 were predicted to have the potential to localise to a eukaryotic nucleus. This included 1399

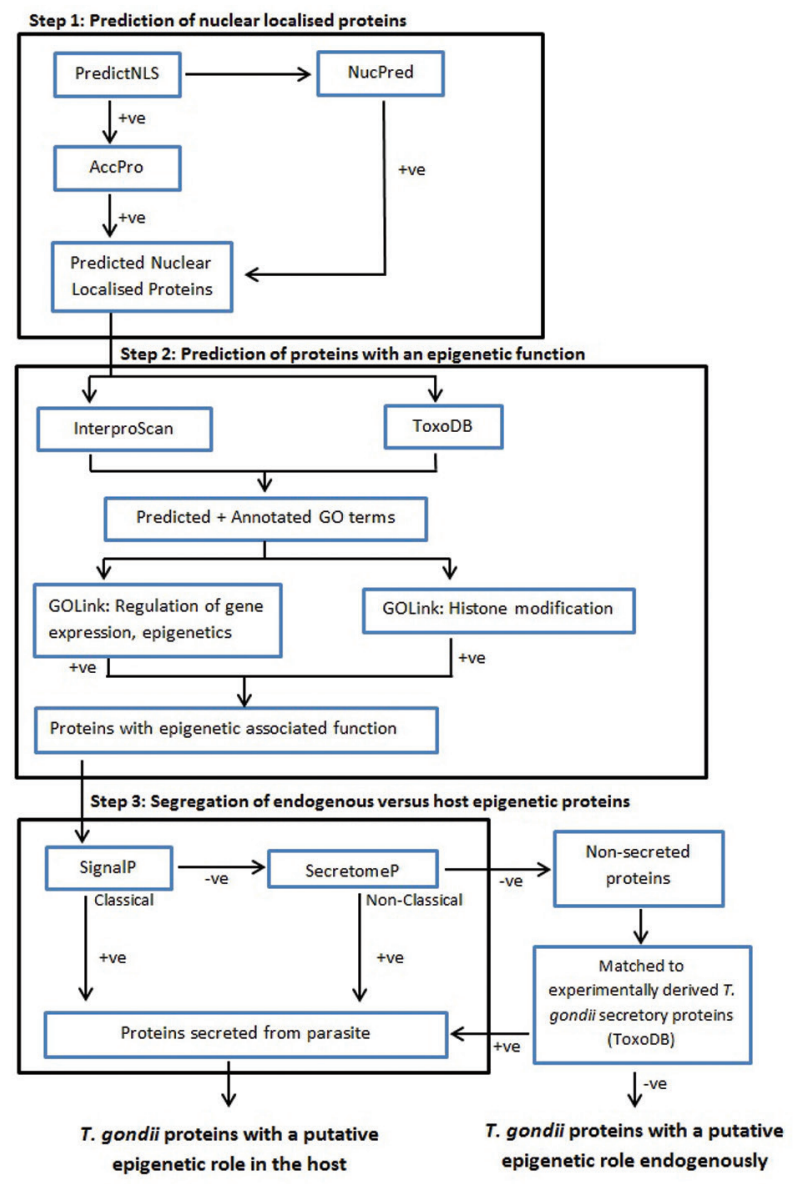

Bioinformatics pipeline outlining the in silico tools and manual methods used to predict parasite proteins with endogenous and exogenous epigenetic function. Step 1: predicts proteins localised to the nucleus; Step 2: predicts proteins with potential epigenetic function; Step 3: applies a filter to determine potential for epigenetic function in the host versus endogenous epigenetic function. proteins that contain a putative functional exposed NLS motif, as predicted by PredictNLS/ACCpro, and 3201 proteins predicted to spend some time in the nucleus by NucPred. There was an overlap of 1192 proteins. The 2009 proteins predicted to be nuclear localised by $\mathrm{Nu}$ cPred alone could contain as yet unidentified NLSs or be chaperoned into the nucleus.

To predict proteins with an epigenetic function we used InterProScan Version 5 (Zdobnov and Apweiler 2001), a tool that allows users to query protein sequences against the InterPro database (Hunter et al. 2012). InterProScan predicts structural motifs and domains and provides any available associated Gene Ontology (GO) terms. We also exported a list of manually annotated and predicted GO terms from ToxoDB (version 8.0) for all T. gondii proteins. To allow inference of epigenetic function from $\mathrm{GO}$ terms across the orthogonal sub-ontologies ("Cellular Component", "Molecular Function" and "Biological Process") we used GOLink (Francis 2013), a tool that collates terms across all sub-ontologies that co-occur with a term of interest across the entire GO database. We used GOLink to compile "terms lists" linked to the query terms "regulation of gene expression, epigenetic" (GO:0040029) and "histone modification" (GO:0016570). We removed the generic GO term "Protein binding" from both lists to reduce false positives. The two GOLink terms lists used in this process can be found at Supplementary data II. All associated GO terms for each protein were then matched to the top 5\% of terms from each of the generated GOLink "terms lists" to determine those parasite proteins predicted to have an epigenetic function. The terms within the 95th percentile were selected as they represent the terms most confidently associated with the initial query terms. Of the 3408 proteins with a prediction of nuclear localisation, 1487 had associated GO terms. The GOLink terms lists (Supplementary data II) obtained for "regulation of gene expression, epigenetic" and "histone modification" contained 140 and 168 terms respectively (representing the top $5 \%$ of all candidate terms returned by GOLink) with an overlap of 68 terms, leaving 240 unique terms that were used in the matching process. Filtering of these 1487 proteins using our GOLink terms lists resulted in 611 proteins (Supplementary data III) that were deemed to be associated with epigenetic functions.

\section{TABLE I}

Summary of proteins filtered through the in silico pipeline. The pipeline was designed to identify a set parasite encoded candidates in the putative secretome that could potentially target the host or parasite nucleus and have domains consistent with an epigenetic function

\begin{tabular}{lcc}
\hline & $\begin{array}{c}\text { Proteins } \\
\text { retained }^{*}\end{array}$ & $\begin{array}{c}\text { Overall percentage } \\
\text { of the proteome }\end{array}$ \\
\hline Initial Toxoplasma gondii proteome & 8318 & $100 \%$ \\
Step 1: prediction of nuclear localised proteins & 3408 & $41 \%$ \\
Step 2: proteins from Step 1 with a predicted epigenetic function & 611 & $7.4 \%$ \\
Step 3a: proteins from Step 2 with a predicted epigenetic function in host & 57 & $0.7 \%$ \\
Step 3b: proteins not included in Step 3a: predicted epigenetic function in parasite & 554 & $6.7 \%$
\end{tabular}

*: protein numbers retained and percent of the entire proteome at each step of the in silico pipeline. 
To identify $T$. gondii encoded proteins that may play a role in modulating the host cell epigenome we used SignalP (version 4.1) to predict proteins with an N-terminal signal peptide (SP) for secretion via the classical secretory pathway. We used the Eukaryote organism groups for our predictions and default D-cutoff values of 0.45 for the SignalP-noTM networks and 0.50 for SignalP-TM networks. Any proteins with a D-score exceeding the relevant cutoff were deemed as having a signal peptide and were included in a putative parasite secretome. As Eukaryotic proteins can be secreted without a classical N-terminal signal peptide, we also used SecretomeP (version 2.0) (Bendtsen et al. 2004) to predict $T$. gondii proteins likely to be secreted via a non-classical secretory pathway. This tool is used in conjunction with SignalP: any protein predicted by both SecretomeP and SignalP is likely to be classically secreted, prediction by SecretomeP only suggests a non-classical mechanism. Proteins with a SecretomeP NN-score $>0.9$ (Bendtsen et al. 2004) were included in the putative parasite secretome. Of the $611 \mathrm{~T}$. gondii proteins with a predicted epigenetic function, 23 were predicted to be secreted via the classical pathway and 1 via the non-classical pathway.

We noted that GRA10 (TGME49_268900) previously shown (Ahn et al. 2007) to be secreted from T. gondii into the host cell and targeted to the host cell nucleus was not predicted to be secreted by either SignalP or SecretomeP. This suggests alternative mechanisms for $T$. gondii proteins to be secreted, for example, the recently reported (Coffey et al. 2015) aspartyl pathway. To address this, the 587 epigenetic candidates with no prediction of secretion by either SignalP or SecretomeP were further screened to determine if any had been proposed to be secreted through experimental studies, in particular the published experimental secretome (Zhou et al. 2005). This yielded an additional 33 epigenetic candidates that were deemed secreted. One caveat in using this experimental secretome data was the observation (Zhou et al. 2005) that a number of cytosolic proteins identified in the secretome could be contaminants due to inadvertent lysis of the parasites during sample preparation. However, since there was no way of distinguishing such false positives from true secreted proteins that might carry novel, possibly $T$. gondii-specific (Coffey et al. 2015), secretory signals we retained the full list of proteins from the experimental secretome in our gene list. This included retaining all proteins that had at least one peptide from the mass spectrometry analysis that mapped to the $T$. gondii database, since there was high probability given the experimental conditions that all peptides identified with a high confidence spectral match to the $T$. gondii genome were of parasite origin. A second caveat was that the experimental secretome contained very few dense granule and no rhoptry proteins, most likely due to the use of $1 \%$ ethanol to stimulate parasite secretion (Zhou et al. 2005). In view of this, the putative secretome list was extended through a search of ToxoDB using keywords such as "Rhoptry", "Dense Granule" and "Microneme".

Overall, 57 proteins were predicted to be secreted and have an epigenetic function (Table II). These proteins represent $0.7 \%$ of the total $T$. gondii proteome and are consid- ered candidates for translocation to the host cell nucleus during infection with a potential role in the manipulation of the host epigenome. The 554 epigenetic candidates not predicted to be secreted were considered to have an endogenous role in the epigenetic regulation of $T$. gondii.

To date, six $T$. gondii secreted proteins have been experimentally shown to target the host cell nucleus. Rhoptry kinase family proteins 16 (ROP16) (Saeij et al. 2007) and 47 (ROP47) (Camejo et al. 2014), protein phosphatase 2C (PP2C-hn) (Gilbert et al. 2007), and dense granular proteins 10 (GRA10) (Ahn et al. 2007), 16 (GRA16) (Bougdour et al. 2013) and 24 (GRA24) (Braun et al. 2013). In addition, we report three proteins that have domains associated with histone modifications: TGME49 207080 (TgMYST-B), which contains a histone acetyltransferase domain; TGME49_202490 containing a histone methylation SET domain; and TGME49 210310 which is predicted to have a histone methylation DOT1 domain. It was previously thought that $T$. gondii did not possess any DOT1 domain histone methyltransferases (Sullivan Jr and Hakimi 2006) but prediction of functional domains using InterProScan now highlights TGME49_210310 as containing a DOT1 domain. The orthologue of TGME49_210310 in the VEG strain of $T$. gondii (TGVEG_210310) is also annotated as having a DOT1 domain in ToxoDB, adding confidence to our prediction of this DOT1 domain in TGME49 210310. Our in silico analysis also identified one protein involved in nucleosome remodelling, TGME49 278440, which contains a SWI/SNF2 related bromodomain. SWI/SNF members are capable of ATPdependent destabilisation of histone-DNA interactions. Finally, we report TGME49_228120, currently annotated in ToxoDB as having a "replication foci targeting sequence" of the type uniquely found in the N-terminal region of DNMT1 molecules (Subramaniam et al. 2014). T. gondii lacks detectable methylcytosine in its DNA (Gissot et al. 2008), and has no full-length DNMT1 homologues. The C-terminal 500 amino acid catalytic portion of DNMTs are conserved between C5 DNMTs of eukaryotes and prokaryotic organisms, and harbour the active centre of the enzyme containing amino acid motifs characteristic of the cytosine-C5-methyltransferase. The N-terminal region of DNMT1 molecules carries 3 sequences, including the replication foci targeting sequence, that increase the precision in copying methylation patterns after DNA replication. As this replication foci targeting sequence is unique to DNMT1 molecules, this suggests that TGME49_228120 may represent the remnants of a $T$. gondii DNMT1 gene.

Overall this in silico analysis has identified several highly interesting candidates for ongoing functional investigations into how $T$. gondii manipulates host cell processes, specifically via the disruption or modulation of the host epigenome. Recently, it has been proposed that distribution of secretory pathogenesis determinants (genes encoding secretory proteins found in MIC, DG, ROP and SRS superfamilies) within the $T$. gondii genome is strain-specific (Lorenzi et al. 2016). Since we have only analysed the ME49 (Type II) proteome, the proteomes of other $T$. gondii strains can be analysed us- 
TABLE II

Proteins from our pipeline with a potential epigenetic function in modulating the host epigenome.

The list is annotated for proteins identified from the in silico secretome, the experimental secretome data, or both

\begin{tabular}{|c|c|}
\hline ToxoDB ID ${ }^{a}$ & Protein description $^{a}$ \\
\hline \multicolumn{2}{|l|}{ In silico secretome } \\
\hline TGME49_207690 & PDCD5 \\
\hline TGME49_213310 & hypothetical protein \\
\hline TGME49_239440 & protein kinase (incomplete catalytic triad) \\
\hline TGME49_241870 & tRNA ligase class I (E and Q), catalytic domain-containing protein \\
\hline TGME49_243280 & Met-10+ like-protein \\
\hline TGME49_245660 & hypothetical protein \\
\hline TGME49_257010 & sporozoite developmental protein \\
\hline TGME49_271625 & serine--tRNA ligase \\
\hline TGME49_277030 & isoleucyl-tRNA synthetase, putative \\
\hline TGME49_281675 & hypothetical protein \\
\hline TGME49_284010 & 5'-3' exonuclease, N-terminal resolvase family domain-containing protein \\
\hline TGME49_295050 & tRNA ligase class II core domain $(\mathrm{G}, \mathrm{H}, \mathrm{P}, \mathrm{S}$ and $\mathrm{T})$ domain-containing protein \\
\hline TGME49_299810 & cysteine-tRNA synthetase (CysRS) \\
\hline TGME49_305920 & endonuclease III family 1 protein \\
\hline TGME49_312370 & RNA pseudouridine synthase superfamily protein \\
\hline TGME49_312520 & tRNA dimethylallyltransferase \\
\hline TGME49_313120 & DNA-directed RNA polymerase, alpha subunit \\
\hline \multicolumn{2}{|c|}{ Experimental secretome } \\
\hline TGME49_202490 & AP2 domain transcription factor AP2VIIa-7 \\
\hline TGME49_206510 & toxolysin TLN4 \\
\hline TGME49_207080 & histone lysine acetyltransferase MYST-B \\
\hline TGME49_210310 & hypothetical protein \\
\hline TGME49_210360 & DEAD (Asp-Glu-Ala-Asp) box polypeptide 41 family protein \\
\hline TGME49_219600 & hypothetical protein \\
\hline TGME49_223880 & zinc finger, $\mathrm{C} 3 \mathrm{HC} 4$ type (RING finger) domain-containing protein \\
\hline TGME49_224480 & cell-cycle-associated protein kinase CLK, putative \\
\hline TGME49_226510 & Sec23/Sec24 trunk domain-containing protein \\
\hline TGME49_228120 & hypothetical protein \\
\hline TGME49_231170 & RecF/RecN/SMC N terminal domain-containing protein \\
\hline TGME49_239420 & protein kinase \\
\hline TGME49_240090 & rhoptry kinase family protein ROP34, putative \\
\hline TGME49_246060 & polymerase (RNA) mitochondrial (DNA directed) POLRMT \\
\hline TGME49_246760 & hypothetical protein \\
\hline TGME49_252500 & polo kinase \\
\hline TGME49_253750 & PLU-1 family protein \\
\hline TGME49_253890 & peptidase M16 inactive domain-containing protein \\
\hline TGME49_267030 & ribonuclease type III Dicer \\
\hline TGME49_268900 & dense granular protein GRA10 \\
\hline TGME49_269885 & rhoptry metalloprotease toxolysin TLN1 \\
\hline TGME49_271290 & hypothetical protein \\
\hline TGME49_271740 & hypothetical protein \\
\hline TGME49_278440 & SWI2/SNF2 Brahma-like putative \\
\hline TGME49_285895 & AP2 domain transcription factor AP2V-2 \\
\hline TGME49_289330 & ubiquitin carboxyl-terminal hydrolase family 2 protein \\
\hline TGME49_292055 & calcium dependent protein kinase CDPK8 \\
\hline TGME49_292235 & hypothetical protein \\
\hline TGME49_294840 & zinc finger (CCCH type) motif-containing protein \\
\hline TGME49_305750 & nucleolar gtp-binding protein 2, putative \\
\hline TGME49_306660 & RNA pseudouridine synthase superfamily protein \\
\hline
\end{tabular}


ToxoDB ID ${ }^{a}$

TGME49_312830

TGME49_313330

Both in silico and experimental secretome

TGME49 201130

TGME49 207610

TGME49 221330

TGME49 229630

TGME49_262730

TGME49 294560

TGME49 309110
Protein description $^{a}$

hypothetical protein

rhoptry kinase family protein ROP27

rhoptry kinase family protein ROP33

rhoptry kinase family protein ROP36 (incomplete catalytic triad)

DNA gyrase/topoisomerase IV, A subunit domain-containing protein eIF2 kinase IF2K-A (incomplete catalytic triad) rhoptry protein ROP16

rhoptry kinase family protein ROP37 (incomplete catalytic triad) tRNA methyl transferase

$a$ : retrieved from ToxoDB (Version 8.0).

ing our freely available pipeline to identify strain-specific proteins that may affect host epigenetics. With the discovery of compounds which act against parasites by inhibiting histone deacetylase (Vanagas et al. 2012), there is increasing interest in identifying the parasite proteins involved in epigenetic regulation, both endogenously and within the host. These proteins represent potential novel drug targets for suppression or treatment of toxoplasmosis.

\section{AUTHORS' CONTRIBUTION}

Conceived and designed the experiments - GS, JMB, RWF and SEJ; performed the experiments and analysed the data GS and RWF; wrote the paper - GS, JMB, RWF and SEJ. The funders had no role in study design, data collection and analysis, decision to publish, or preparation of the manuscript.

\section{REFERENCES}

Ahn H-J, Kim S, Nam H-W. Nucleolar translocalization of GRA10 of Toxoplasma gondii transfectionally expressed in HeLa cells. Korean J Parasitol. 2007; 45(3): 165-74.

Bendtsen JD, Jensen LJ, Blom N, Von Heijne G, Brunak S. Featurebased prediction of non-classical and leaderless protein secretion. Protein Eng Des Sel. 2004; 17(4): 349-56.

Bougdour A, Durandau E, Brenier-Pinchart M-P, Ortet P, Barakat M, Kieffer S, et al. Host cell subversion by Toxoplasma GRA16, an exported dense granule protein that targets the host cell nucleus and alters gene expression. Cell Host Microbe. 2013; 13(4): 489-500.

Brameier M, Krings A, MacCallum RM. NucPred - Predicting nuclear localization of proteins. Bioinformatics. 2007; 23(9): 1159-60.

Braun L, Brenier-Pinchart M-P, Yogavel M, Curt-Varesano A, CurtBertini R-L, Hussain T, et al. A Toxoplasma dense granule protein, GRA24, modulates the early immune response to infection by promoting a direct and sustained host p38 MAPK activation. J Exp Med. 2013; 210(10): 2071-86.

Camejo A, Gold DA, Lu D, McFetridge K, Julien L, Yang N, et al. Identification of three novel Toxoplasma gondii rhoptry proteins. Int J Parasitol. 2014; 44(2): 147-60.

Coffey MJ, Sleebs BE, Uboldi AD, Garnham A, Franco M, Marino $\mathrm{ND}$, et al. An aspartyl protease defines a novel pathway for export of Toxoplasma proteins into the host cell. Elife. 2015; 4: pii: e10809.

Cokol M, Nair R, Rost B. Finding nuclear localization signals. EMBO Rep. 2000; 1(5): 411-5.
Francis RW. GOLink: finding cooccurring terms across gene ontology namespaces. Int J Genomics. 2013; 2013: 594528.

Gajria B, Bahl A, Brestelli J, Dommer J, Fischer S, Gao X, et al. ToxoDB: an integrated Toxoplasma gondii database resource. Nucleic Acids Res. 2007; 36(Database Issue): D553-D6.

Gilbert LA, Ravindran S, Turetzky JM, Boothroyd JC, Bradley PJ. Toxoplasma gondii targets a protein phosphatase $2 \mathrm{C}$ to the nuclei of infected host cells. Eukaryot Cell. 2007; 6(1): 73-83.

Gissot M, Choi S-W, Thompson RF, Greally JM, Kim K. Toxoplasma gondii and Cryptosporidium parvum lack detectable DNA cytosine methylation. Eukaryot Cell. 2008; 7(3): 537-40.

Hettmann C, Soldati D. Cloning and analysis of a Toxoplasma gondii histone acetyltransferase: a novel chromatin remodelling factor in Apicomplexan parasites. Nucleic Acids Res. 1999; 27(22): 4344-52.

Hunter S, Jones P, Mitchell A, Apweiler R, Attwood TK, Bateman A, et al. InterPro in 2011: new developments in the family and domain prediction database. Nucleic Acids Res. 2012; 40(Database Issue): D306-D12.

Lang C, Hildebrandt A, Brand F, Opitz L, Dihazi H, Luder CG. Impaired chromatin remodelling at STAT1-regulated promoters leads to global unresponsiveness of Toxoplasma gondii-infected macrophages to IFN-gamma. PLoS Pathog. 2012; 8(1): e1002483.

Leng J, Butcher BA, Egan CE, Abdallah DS, Denkers EY. Toxoplasma gondii prevents chromatin remodelling initiated by TLR-triggered macrophage activation. J Immunol. 2009; 182(1): 489-97.

Leng J, Denkers EY. Toxoplasma gondii inhibits covalent modification of histone $\mathrm{H} 3$ at the IL-10 promoter in infected macrophages. PLoS ONE. 2009; 4(10): e7589.

Lorenzi H, Khan A, Behnke MS, Namasivayam S, Swapna LS, Hadjithomas $M$, et al. Local admixture of amplified and diversified secreted pathogenesis determinants shapes mosaic Toxoplasma gondii genomes. Nat Commun. 2016; 7: 10147.

Magnan CN, Baldi P. SSpro/ACCpro 5: almost perfect prediction of protein secondary structure and relative solvent accessibility using profiles, machine learning and structural similarity. Bioinformatics. 2014; 30(18): 2592-7.

Saeij JPJ, Coller S, Boyle JP, Jerome ME, White MW, Boothroyd JC. Toxoplasma co-opts host gene expression by injection of a polymorphic kinase homologue. Nature. 2007; 445(7125): 324-7.

Sautel CF, Cannella D, Bastien O, Kieffer S, Aldebert D, Garin J, et al. SET8-mediated methylations of histone H4 lysine 20 mark 
silent heterochromatic domains in apicomplexan genomes. Mol Cell Biol. 2007; 27(16): 5711-24.

Subramaniam D, Thombre R, Dhar A, Anant S. DNA methyltransferases: a novel target for prevention and therapy. Front Oncol. 2014; 4: 80.

Sullivan Jr WJ, Hakimi M-A. Histone mediated gene activation in Toxoplasma gondii. Mol Biochem Parasitol. 2006; 148(2): 109-16.

Sullivan Jr WJ, Monroy MA, Bohne W, Nallani KC, Chrivia J, Yaciuk $\mathrm{P}$, et al. Molecular cloning and characterization of an SRCAP chromatin remodelling homologue in Toxoplasma gondii. Parasitol Res. 2003; 90(1): 1-8.

Syn G, Blackwell JM, Jamieson SE. Chapter 19. Epigenetics in infectious diseases. In: JL Garcia-Gimenez, editor. Epigenetic biomarkers and diagnostics. Amsterdam, Boston, Heidelberg, London, New York, Oxford, Paris, San Diego, San Francisco, Singapore, Sydney, Tokyo: Elsevier Inc and Academic Press; 2016; p. 377-94.
Vanagas L, Jeffers V, Bogado SS, Dalmasso MC, Sullivan Jr WJ, Angel SO. Toxoplasma histone acetylation remodelers as novel drug targets. Expert Rev Anti Infect Ther. 2012; 10(10): 1189-201.

Wang J, Dixon SE, Ting L-M, Liu T-K, Jeffers V, Croken MM, et al. Lysine acetyltransferase GCN5b interacts with AP2 factors and is required for Toxoplasma gondii proliferation. PLoS Pathog. 2014; 10(1): e1003830.

Wei H, Jiang S, Chen L, He C, Wu S, Peng H. Characterization of cytosine methylation and the DNA methyltransferases of Toxoplasma gondii. Int J Biol Sci. 2017; 13(4): 458-70.

Zdobnov EM, Apweiler R. InterProScan - an integration platform for the signature-recognition methods in InterPro. Bioinformatics. 2001; 17(9): 847-8.

Zhou XW, Kafsack BF, Cole RN, Beckett P, Shen RF, Carruthers VB. The opportunistic pathogen Toxoplasma gondii deploys a diverse legion of invasion and survival proteins. J Biol Chem. 2005; 280(40): 34233-44. 